\title{
Article
}

\section{Insider Action research as an approach and a method - Exploring institutional encounters from within a birthing context}

Nyman, Viola, Berg, Marie, Downe, Soo and Bondas, T

Available at http://clok.uclan.ac.uk/12450/

Nyman, Viola, Berg, Marie, Downe, Soo ORCID: 0000-0003-2848-2550 and Bondas, $T$ (2015) Insider Action research as an approach and a methodExploring institutional encounters from within a birthing context. Action Research . pp. 1-17. ISSN 1476-7503

It is advisable to refer to the publisher's version if you intend to cite from the work. http://dx.doi.org/10.1177/1476750315600225

For more information about UCLan's research in this area go to http://www.uclan.ac.uk/researchgroups/ and search for <name of research Group>.

For information about Research generally at UCLan please go to http://www.uclan.ac.uk/research/

All outputs in CLoK are protected by Intellectual Property Rights law, including Copyright law. Copyright, IPR and Moral Rights for the works on this site are retained by the individual authors and/or other copyright owners. Terms and conditions for use of this material are defined in the policies page. 


\title{
Insider Action research as an approach and a method - Exploring institutional encounters from within a birthing context
}

Action Research 0 (0) $1-17$

(C) The Author(s) 2015

Reprints and permissions: sagepub.co.uk/journalsPermissions.nav DOI: I0.I|77/|4767503 |5600225 arj.sagepub.com

(SAGE

\section{Viola Nyman}

Institute of Health and Care Sciences, Sahlgrenska Academy, University of Gothenburg, Gothenburg, Sweden

\section{Marie Berg}

Institute of Health and Care Sciences, Sahlgrenska Academy, University of Gothenburg, Gothenburg, Sweden; Centre for Person-Centred Care (GPCC) University of Gothenburg, Gothenburg, Sweden; Sahlgrenska University Hospital, Gothenburg, Sweden

\section{Soo Downe}

University of Central Lancashire, School of Health, Preston, UK

\section{Terese Bondas}

University of Nordland, Faculty of Professional Studies, Bodö, Norway

\begin{abstract}
The aim of this paper was to describe the first person perspective of being a peer midwife and a novice researcher initiating collaborative AR in her own organization to develop knowledge about the first encounters between the labouring woman and her care-givers in a hospital birthing context. It was motivated by the author's longstanding professional clinical experience of observing and hearing parents' stories of vulnerability and fear of childbirth, and how staff's attitudes affected the childbirth experience negatively. Data were collected between 2010 and 2013 and included the researcher's log with reflections from clinical work, as well as interviews, participant observation, and research group communications. A reflective interpretative lifeworld research approach
\end{abstract}

\section{Corresponding author:}

Viola Nyman, Institute of Health and Care Sciences, Sahlgrenska Academy, University of Gothenburg, Box 457, Gothenburg SE-405 30, Sweden.

Email: viola.nyman@vgregion.se 
was used to analyze the data. The experience of being a novice insider action researcher (IARr) consisted of three thematic meanings: "the struggle to initiate a clinical insider action research project," "standing alone at the messy front line," and "being a catalytic counterbalance to the prevailing medico technical focus." The comprehensive understanding was "learning how to clinically reflect on and to voice the tacit components of care." The strategy used in undertaking this study was influenced by the philosophies of both midwifery care and AR.

\section{Keywords}

Insider action research, health services research, midwifery, caring, institutional encounters, organizational development

\section{Introduction}

The purpose of this paper is to describe a novice IARr's activities in relation to the initiation of an Action Research (AR) study. The study was set up to develop knowledge about, and to improve the experience of the first encounter between birthing couples and the midwives they meet when they arrive in hospital in labour. Implementing change in health care is complex, and the results are often short lived (Hellström, Lifvergren, \& Quist, 2010; Parkin, 2010). This may be partly because change is often introduced in a top-down manner. AR has been used within health organizations as a useful way of democratizing change through research activities (Waterman, Tillen, Dickson, \& de Koning, 2001) and of promoting health-care development (Meyer, 2000; Munn-Giddings, McVicar, \& Smith, 2008). Epistemologically, AR is concerned with changing the world as well as describing and explaining it (Reason \& Bradbury, 2006). It takes into account the researchers' and participants' experiences and opinions, and aims to bring together action, reflection, theory and practice (Hart, 1995; McNiff \& Whitehead, 2011; Reason \& Torbert, 2001; Shani, Pasmore, \& Woodman, 2012; Winter, Munn-Giddings, \& Atmer, 2001). AR was therefore used as a vehicle for change within a particular maternity care setting in Sweden. As part of the study, reflexive accounting took place, to try to understand how AR worked and did not work in this specific context. Doing an AR in your own organization and in your own unit not only as a professional but also as a doctoral student is complex and demanding. The paper aims to illuminate these challenges.

The study was motivated by the first author's long clinical experience as a midwife, including her counseling work with women and partners who had fear of childbirth, and from having interviewed obese women about their experiences of encounters with the maternity care. These service users recounted stories of how staffs behaviours and attitudes affected their childbirth experience negatively. 


\section{Insider action research and midwifery}

Undertaking IAR implies a process of inquiry as a permanent member of an organizational system (Adler, Shani, \& Styhre, 2004; Coghlan \& Brannick, 2009). It usually requires both an academic and an organizational perspective. The range of possible study designs includes planned insider/outsider teams, where organizational members and academic researchers are involved in every stage of the process; being an IARr who acts as a facilitator for the interaction between insiders and outsiders; or separate teams where the IARr is an active member of both constituents (Adler et al., 2004). In the study presented in this paper, the latter approach was used. One challenge of doing first person action research is how to gain feedback. The response from colleagues, and the degree to which the field of issues in which the AR is engaged, is open to change, and might be circumscribed by the relationship the insider researcher wants to explore (Marshall, 2004). This is especially acute when the IARr is a professional, with the moral, ethical and professional requirement to actively prioritize the wellbeing of service users, and with the ongoing need to maintain a positive relationship to professional peers, at the same time as undertaking the research study.

AR has been used previously by midwife researchers. Barrett (2005) studied use of ordinary talk before and after childbirth, with the aim of improving midwifery practice in this area. Deery (2005) explored community midwives' views and experiences of their support needs in clinical practice. Mander, Cheung, Wang, Fu, and Zhu (2010) explored the possibility of a midwife-led normal birthing unit in mainland China to create a more suitable environment for supportive care. Russell (2011) investigated the variations in the availability of water birth on one hospital labour ward. There are also a numerous accounts of AR studies undertaken by nurses. However, our literature search did not identify any publication written from the first person perspective of being a peer midwife and a novice researcher initiating AR in her own organization with the simultaneous ambition of developing knowledge for practice change. This paper therefore adds to the current literature by presenting a reflexive account of experiences during the process of undertaking IAR.

\section{Preunderstanding: A caring relationship is a prerequisite in childbirth care}

Before the AR study commenced, the IARr (VN) and the external research group (MB, SD and TB) believed that establishment of a caring relationship was fundamental for developing optimal care in childbirth (Berg, Ólafsdóttir, \& Lundgren, 2012; Hunter, Berg, Lundgren, Ólafsdóttir, \& Kirkham, 2008; Kirkham, 2010). A caring relationship is grounded in the philosophical objective of the human having a responsibility for the other, or in the words of Levinas "not letting the other alone" (Lavoie, De Koninck, \& Blondeau, 2006). People need to meet each 
other authentically in a direct subject-subject interpersonal relationship of mutuality and reciprocity where they share a sense of caring, respect, commitment, and responsibility (Buber \& Kaufmann, 1970). In order to cope with labour effectively, it is essential that the laboring woman feels that midwives and other caregiver(s) are present both physically and mentally. This "presencing" includes being seen as a person, having a trusting relationship, being supported and guided on one's own terms (Berg, Lundgren, Hermansson, \& Wahlberg, 1996), and for the midwife to handle being present, and to do "nothing" well when the labour is proceeding smoothly (Kennedy, 2000). From a longer term perspective, childbirth is also a potentially life-changing experience, when women can encounter new aspects of their personhood and that of their accompanying partner (Lundgren, Karlsdottir, \& Bondas, 2009). This liminal process can be enabled by good quality midwifery care that is authentically woman focused. However, for a range of reasons, including the culture and values of the maternity services, power asymmetry between staff and their colleagues, and between women and staff, and the spread of technocratic and risk focused care provision, such care is not always provided (O'Connell \& Downe, 2009). The design and conduct of our research project was predicated on, and influenced by, this set of factors.

\section{The aim of this paper}

The aim of this paper is to describe an insider action researcher's experiences as a peer midwife and a novice researcher doing action research collaboratively to develop theory and practice in the first encounter on a labour ward.

\section{The AR process}

In AR, the selected research topics often are carried out as part of ordinary work rather than as a separate exercise, since there is an expectation that each AR project will make a useful contribution to the organization (Coghlan \& Brannick, 2009). For VN, this research journey started in 2008 after undertaking a study of obese women's experiences of encounters with staff. That study highlighted the need for staff to verbalize personal prejudices, not only about obesity but also about the optimal care approach in all encounters on the labour ward. It initiated an aspiration to both improve the local approach to care and to increase knowledge about caring science. In 2009, the service for women who had tocophobia (fear of childbirth) at the study research site was downsized due to economic constrains. VN had earlier worked in this service and heard women and partners talk about their fears relating to labour and birth, and, specifically, carers' behaviours. This raised a question about how to improve encounters between women and staff by extending the principles of the service that had previously only been offered to women with fear of childbirth. This entailed a conscious effort to attentively listen to the stories and needs of all women and their partners. The first issue to address was to find out how parents had experienced the first encounter on the local labour ward. 
These initial studies of encounters led to the question of how to approach the issue of enhancing the quality of encounters in the local organization.

\section{The Normal Labour Process project within which the AR study was nested}

The setting for the AR study was a labour ward at a hospital in Sweden where the local Regional Council had introduced a systematic quality development project, "Care 2010" in accordance with the national Swedish governmental recommendations (2005: 12). The objective of the "Care 2010" project was to introduce a quality development model through which each clinic within the organization would establish goals, and follow them, based on a structured approach. The overall intention was to decrease the time needed for each hospital stay and to create a basis for continuous quality development in the future. Following the request from the local Regional Council, the Head of the participating maternity unit indicated support for a quality development project on normal labour. The project was designed to include women entering the labour ward with an expected vaginal birth. The intervention was titled the "normal labour process (NLP)." The AR study at the centre of the current paper was nested within the project. At the outset of the AR study, there were 50 midwives and 20 health-care assistants employed on the labour ward. Midwives were the primary providers of care of normal labour and birth. Consultations with physicians took place if complications in labour occurred during birth, but the physicians were not based on the ward. The first author (VN), in two of the three simultaneous roles noted above, was both a full member of the organization and a doctoral student. She was one of seven staff members, five midwives and two health-care assistants, who voluntarily joined the group for the NLP project. One physician was also supposed to participate, but after a couple of meetings she moved and no other physician joined the group. A clinical NLP group was formed, with the first author being chosen by the group members to be the process leader. The AR element of the NLP was carried out for the period of four years; one year of clinical collaborative planning for action, followed by three years of action, evaluation and re-planning iteratively.

For the AR component, an external research group was set up, which consisted of the IARr (VN) and three senior researchers (MB, SD and TB), who also acted as supervisors and as such two of them made visits to understand the context, and to support the IARr.

\section{One action leads to another}

Through the mapping of the course of normal labour, one action led to another and many different cycles were formed more or less concurrently, emphasizing not only the first encounter but also the need to optimize the general care approach and routines. Additionally, the processing enabled many small practical changes initiated by the NLP group and implemented by staff prior to the implementation 
of the emphasized first encounter. Based on the dialogues with colleagues, the IARr was able to formulate their ideas alongside her own interpretations, to plan, do and evaluate actions for quality improvement. The IARr also undertook several actions in the doctoral student role, separately from the regular NLP group communication with staff, some which are described in table one, which also shows the timing of the events, and thus how they developed iteratively over time. The following bullet points offer more detail on some of the events listed in Table 1, as examples of how the process evolved:

- 2009-2010: Starting in October 2010 with a brainstorm on a staff meeting about what to focus in the first encounter, the primary interest of the IARr. An attempt to awake reflection by inviting staff to write good birth stories was unsuccessful because no stories were produced. In a subsequent attempt to stimulate reflection, sheets with quotes relating to the experiences of women and partners' who had taken part in the first encounter study were disseminated to staff. For example, some quotes illustrated that the compulsory admission CTG (electronic fetal monitoring) led to unnecessary suffering for some women in labour. One father who was not happy with the procedure said: "Just this, the silence or, entering the room and put on the CTG and then leave" (FG1P) (Nyman, Downe, \& Berg, 2011).

- 2011: Transcribed individual interviews with 37 colleagues (four of the interviewees were also members of the NLP group) were held in the spring of 2011 to explore beliefs, views and experiences relating to their first encounter with women and partners, and to create reflection (Nyman, Bondas, Downe, \& Berg, 2013). This replaced the original plan, to have focus groups, which failed because it was impossible to assemble midwives together all at the same time, due to time constraints.

- 2011-2012: As the AR project progressed, midwives started to extend their reflections from the first encounter, to other aspects of normal labour, including debate at staff meetings about the need for various routine interventions decided by midwives and/or physicians. An evaluation of women's and partners' experiences of childbirth including the first encounters through a web-based survey was planned but had to be postponed because it had a low response rate. Instead the focus turned to (unnecessary) interventions in normal labour, including an assessment of whether these rates had decreased due to the emphasis on improving care. This inquiry led to an emphasis on discussing interventions in routine management of labour, specifically, duration of admission CTG (electronic fetal heart monitoring), amniotomy (breaking the waters), use of fetal scalp electrode, and augmentation of labour with oxytocin. These issues will be described elsewhere.

- 2013: The final evaluation process in the AR study was focus groups with staff. An attempt to do this a year earlier failed because it was not timely, for many organizational reasons. Finally, in 2013, before completing data collection and starting the final write up of the doctoral thesis, the focus discussions were held. 


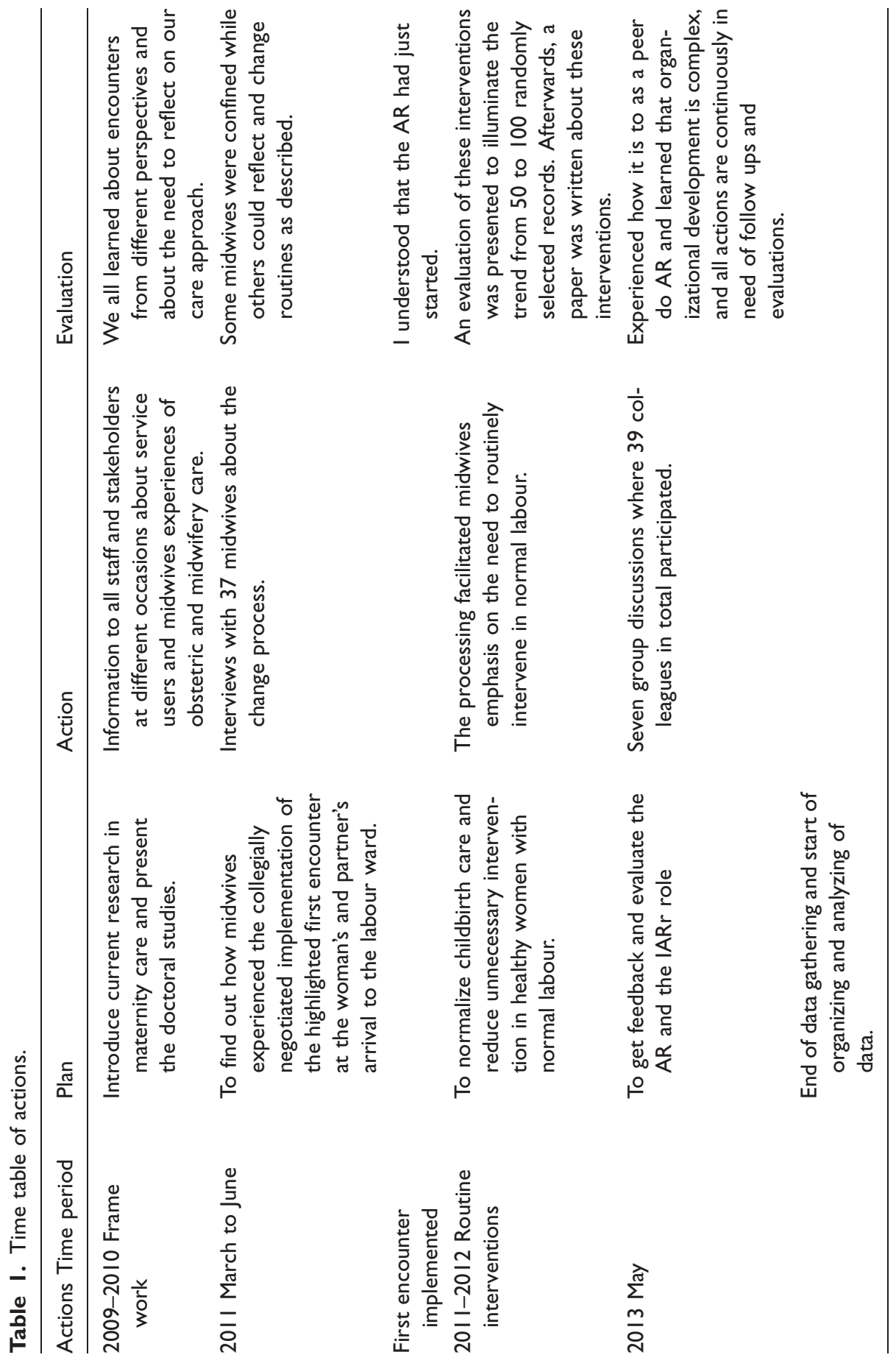




\section{Method}

\section{Ethical considerations}

The ethical principles based on the Helsinki declaration guided this research involving human subjects, in accordance with Brydon-Miller's (2008) key ethical principles in AR; respect for persons, beneficence, and justice as shared values of a democratic process of engagement in morally committed actions. As a peer, familiar with the system's culture and beliefs, it was important to distinguish how and what information to disclose relating to others and self. It is necessary for insider researchers to critically examine their own core values (Brydon-Miller, 2008). The process and its consequences are further illuminated in this paper. The senior authors have carried out AR and intervention studies previously, but neither the IARr nor any of the other authors had any prior experience of being an IARr, and so they were encountering these specific ethical issues for the first time in the course of this study.

\section{Data collection}

The data that form the basis of the reflexive account given in this paper were collected iteratively and informally between 2010 and 2013, as the AR process unfolded. This was congruent with what has been described by Adler et al. (2004) who stated that in collaborative operational research (such as AR), there is no generically viable toolbox. Data for this paper consisted of the IARr's log (personal notes) including reflections from clinical work, communication with the external research group and interviews with colleagues to judge timeliness for actions (Chandler \& Torbert, 2003).

\section{Data analysis}

A reflective interpretative life world research approach was used to discover qualitative patterns of meanings, comprehension and explanations of the studied phenomenon (Dahlberg, Dahlberg, \& Nyström, 2008). This is an inductive interpretative approach without a predetermined hypothesis. The approach is based on the philosophical underpinning that description and interpretation is a key for understanding the essence of phenomenon in daily life (Gadamer, Weinsheimer, \& Marshall, 2004) and that sensitivity about, and flexibility to the studied phenomenon based on the researcher's experience is crucial (Dahlberg et al., 2008).

The analysis of the data aimed at discovering tentative qualitative meanings in the text, which were then synthesized into a comprehensive interpretation of the phenomenon. First, all the text form field notes was read through to get a sense of the whole. This included diverse small evaluations, and reflections on the whole process and on previous research. Next, in further readings, an intensive dialogue with the text was performed where meaning units were identified and clustered. 
Following the hermeneutic circle, the whole of the data text was interpreted in terms of the details, and the details were then examined in terms of the whole. Successively, themes of meaning were recognized and described. These formed a basis for a final whole description and comprehensive interpretation of the explored phenomenon (Dahlberg et al., 2008).

The first analysis was performed by the IARr, and subsequent analysis and interpretation was performed by all authors. This resulted in two levels of meaning, totalling four themes: three themes at the first level and then a final comprehensive interpretation. An auto-ethnographic writing style is used for the first level themes of meaning, to take account of direct engagement with the data as one of the many ways of knowing and inquiring within the world (Heron, 1996; Reason \& Torbert, 2001). This allows for the telling of a personal narrative (Ellis \& Bochner, 2000) as a type of critical enquiry into the practice of a researcher and/or practitioner (McIlveen, 2008). In keeping with this intent, the personal pronoun is used in the findings section of the paper. In the comprehensive interpretation, a theoretically generalizable understanding of the phenomenon, the collective pronoun is used.

\section{Results}

The essence of the experience of being a clinically qualified IARr consisted of three thematic meanings: "the struggle to initiate a clinical insider action research project," "standing alone at the messy front line," and "being a catalytic counterbalance to the prevailing medico technical focus." The comprehensive understanding of the phenomenon was "learning how to clinically reflect on and to voice the tacit components of care."

\section{The struggle to initiate a clinical insider action research project}

The task taken on by the NLP group (mapping the pathway of normal labour) was a major undertaking, and directions from management were not clear. This resulted in uncertainty for me and significant frustration for all of us in the NLP group during the first year. This is shown in the following quote from one of the members:

In the beginning it was unclear for me what was your (VN's) research and what was the process, it was a bit messy for me and maybe it was because I thought that everything took too long time, we did not focus on what we were supposed to,... it goes hand in hand the first encounter and so, but I thought, are we going to continue like this that we both have the research and the process work then it will be difficult, but it have worked really well. ("Normal labour process" group member, 28 March 2011)

I was frustrated by the slow and invisible progress. Many days I wondered if taking an AR approach was a good idea after all, as it was so slow and such a 
testing experience, but, on other days, it felt right, and the most appropriate explication for the question I was addressing. These days allowed me to catch when staff was receptive to talk about women's and partners experiences of the first encounter. Nevertheless, doing AR felt for me like an assignment I was supposed to do on the side, when there was time, despite the fact that AR is supposed to be a democratic process. In the beginning, I was unsure who my insider group was and who was my external research group? Which group did I belong to? AR was new to everybody, including me, and it took a while for me to learn that the "normal labour process" group, though set up for the larger project, could also be the insider research group for this AR study. By the time I formally asked the group if they wanted to be the co-researchers, they were already actively engaged in this role. The question of participation haunted me. How participative was the staff, in fact? This led me to feelings of personal fault and failure and was an issue continuously discussed in the supervision sessions. Through the years of the study, I asked myself questions such as Is the way this project was initiated a reason for its fuzziness? How could we have done this differently? What will I do next? How can we make this processing more democratic? Is this AR at all? Is this AR experienced as top down?

\section{Standing alone at the messy front line}

The spring of 2012 was a turbulent time for the staff due to high workloads, shortage of staff, and an organizational change around lunch breaks. This anxiety and frustration also affected the dynamics of the group. Data in the IAR study collected around this time revealed that the addition of the AR study to the NLP project was felt to be an extra and unwanted burden, as described in the following quote from one of the NLP members:

When we started this process we did not have your research at all in our heads, so I thought that the research took too much time from the processing, but we have seen that it had added things also. Not saying that it is unimportant, but we have limited time. ("Normal labour process" group member, 5 May 2012)

I did not feel understood or supported at all, but I just had to stay put, wait and see what would happen, even if it did not feel like I wanted to stay at all. This was the peak point where I really wanted to quit the research, but being an employee, you do not easily just give up and leave. To give up would be to fail totally. I thought about a couple of other action researchers who had quit doing AR due to the fuzziness, probably at this stage. About two years had gone by, and it felt like very little was coming out of my work.

Through my own self-reflection, I got closer to understanding the causes of my anxiety about the study. Specifically, I became aware of my fear of failure; both failure in succeeding with my ambitions of improving the local approach to maternity care and failure in managing to complete my doctoral studies. This resulted in 
distress about being perceived as incapable by colleagues or stakeholders, a feeling an external researcher would probably not have due to lack of the enduring bonds to the participants.

\section{Being a catalytic counterbalance to the prevailing medico technical focus}

Working as a clinical midwife, I reflected that the only visible change in terms of the first encounter was that women were on the electronic foetal monitoring system for a shorter time than before the project started. As a consequence, I presented regular feedback to staff about what happened in the research process, including papers about midwifery care to staff and other stakeholders in all kinds of suitable situations. Midwives started to discuss "normal labour" more regularly, including staff meetings, as the following extract from a set of staff meeting notes reveals:

Information is available on the intranet about the "normal labour process" and in the folder, please read the report from week 47. There is a "Problem list" where everybody can write about things they want the group to look at. Some reflections below. - The first meeting dialogue is important for the women and partner, and for ourselves, it also decreases the time the admission CTG runs. In the beginning of 2012 the trend was getting back to long admission CTG traces, but the feeling now is that we have improved.

- We use less scalpelectrodes routinely than earlier, from 1.3 electrodes to 1 per labour. We have also decreased performing amniotomy and starting Oxytocin. (Minutes from staff meeting, 12 December 2012)

I was also positively surprised that, as colleagues, we began to spontaneously discuss our own behaviours in the first encounter and elsewhere, and to talk about the caring perspective of our profession. For me this meant that change had happened. New questions appeared in my head: does this mean that, now, midwives have more time to be "truly present" in the first encounter? My intention was never to be in the rooms to observe the first encounter between the midwife and couple, and so I could not directly verify this hypothesis. As well as more discussion about normal birth, extreme risk-averse thinking and an excessive focus on problems and potential pathology were regularly noticed and confronted. This was a clear and positive result of the project. The initial focus of improving the first encounter was catalytic for staff to reflect on the whole labour process.

For me, evidence that the intention of the AR study was realized became clear in spring 2013 in seven focus group sessions with staff where I inquired about their experience of participating in this AR and of me as a researcher. They had difficulties in telling me about my role as the IARr (as I did in asking about it) other than encouraging me to continue. However rather to my surprise, staff said about the first encounters "we have always thought that the first encounters are 
important." This is not, in fact, reflected in the data I collected at the beginning of the study; however, it reassured me that the specific focus chosen was appropriate.

\section{Comprehensive interpretation: Learning how to clinically reflect on and voice the tacit components of care}

The themes illustrate how the IARr became an active agent "struggling to initiate clinical insider action research" to create space in which staff could consider the importance of, and techniques for, responding to the women's and partners' needs in their first encounter with staff on the arrival on a labour ward. A feeling of "standing alone at the messy front line" included a process of adjusting oneself, and of searching to find a balance between being an insider and an outsider, including acceptance of the inherent and physical chaos embedded in doing the IAR project. Over time, it became clear that AR was the appropriate way of improving the care elements that were identified for change. Crucially, it became very apparent that this kind of action takes time; it is not possible to have a quick solution or rapid practice change. It became also apparent that the four years of negotiations about normalizing labour starting with the first encounter meant that the IARr was the catalyser for a counterbalance to the prevailing medico technical focus of care. This led to the conclusion that the culture might be beginning to change. Yet, it required far more than a simple protocol change or technical fix. To change care (including practical) routines on the labour ward is to change fundamentally how it is to be a professional carer. It is to take the responsibility for the caring relationship by "not letting the Other alone" (c.f. Levinas).

\section{Discussion}

In this study, the phenomenon of being a clinical nurse midwife and a novice IAR can be summarized by the process of learning how to clinically reflect on and to voice the tacit components of care through a first person perspective. The original research project was set up as a consequence of caring about a fellow human's emotional comfort in any (dependent) situation and specifically to support people in the new crucial life event of childbirth. This follows the ontological assumption of AR that it is morally and ethically important to try to live in a way consistent with one's own values (McNiff \& Whitehead, 2011).

The IARr (VN) was from the start influenced by two potentially conflicting world views. On the one hand, she was integrated into the prevailing risk-focused labour ward culture of the study site. On the other hand, she understood the emotional and psychosocial stress that this culture generated for labouring women and their partners, especially when they first encountered the ward. Organizational politics implies balancing the requirements for the success and quality of the AR, future employment, and maintaining credibility as an effective driver of a decided topic of focus (Coghlan \& Brannick, 2009). Earlier improvement projects in the study site had been more top-down, where the IARr has been a 
participant in research and specific interventions, usually obstetric or medical improvements initiated and controlled by others. The difference for this AR project is that it involved intangible actions when spending time to build a relationship with the mother and her partner, additionally to the reduction of clinical routine procedures. Paradoxically, in a so-called caring health system, this was counterintuitive: doing less tasks and more personal engagement was seen as deviant by some staff, usually due to time constraints. In fact, according to Hoag, Ritschard, and Cooper. (2002), change is not usually dependent on lack of time but instead on lack of will. Decisions that change the system and individuals are constantly taken and occur naturally anyway, for example, through staff turnover, or the introduction of new products or services. It is what individuals choose to change at any specific time that is decisive (Hoag, et al. 2002).

To be a change agent, and a colleague, and a researcher risks role duality, or role conflict (Holian \& Coghlan, 2012). The reflexive accounting presented in this paper provides an account of how these roles influenced VN's self-view of the world, as well as how she was perceived by others involved in the AR process (Coghlan \& Brannick, 2009). It required VN to reflexively and actively try to maintain credibility in the eyes of the colleagues, both to achieve the planned change in itself and to be able to continue the research study (Nugus, Greenfield, Travaglia, \& Braithwaite, 2012). It may be thought easier to do IAR as a peer, posing less of a threat to disturb the hierarchical order or "upset the equilibrium" as Moore (2007) experienced doing insider research in his manager position. As a peer the IARr did not pose a threat to the hierarchy, but the staff relied on the IARr to take the responsibility for the project's successes and failures.

It seems that the vehicle for this more tacit, and, potentially, more sustainable change in attitudes was the spontaneous discussions during the years that took place between staff in relation to the changes instituted by the AR study. The ordinary institutional talks that develop an emotional and social care approach (Deery, 2005) are potentially available in health care as part of the essential communications that take place between peers as part of professional practice. From the beginning, the change in daily routines was discussed within these ordinary professional encounters, with the ambition to improve care. The iterative nature of these kinds of conversations indicates unpredictability inherent in any attempt to clearly identify the focus of change and in the expected results in AR projects (McVicar, Munn-Giddings, \& Abu-Helil, 2012).

In organizations and health systems, the ethical and political challenge for action researchers is to deliver straightforward feedback that can engage both frontline staff and managers (Nugus et al., 2012) and to generate knowledge for the academy (Coghlan, 2013). An IARr needs to be both close to the setting, and also to be able to create distance from it to be able to see things critically and thus enable change to happen (Coghlan \& Brannick, 2009). To be both close and distant in order to perform effectively was a challenge for VN, especially as she was working with known colleagues for more than 20 years. Reflection on the interaction between an IARr and study participants is said to illuminate how changes occur 
(Chiu, 2006). To describe the IAR's experiences as a peer midwife and a novice researcher made the reflection complex and confusing. When writing about the process, it was hard to avoid getting lost in the messy reality of the real lifeworld and practice, and to determine what to choose to tell from the text. Similarly Heen (2005) searched for words and expressions for something she wanted to communicate without knowing what to write when being involved in IAR. It is like being immersed in the "swampy lowlands" (Schön, 1995) with the feeling that trying to get up to higher ground to study and describe from a distance is almost an unmanageable mission. Yet, in this case, the problem was surmountable with support and frequent correspondence between the co-authors. Under any conditions, doing IARr as a doctoral student can be recommended because of the possibility one gets to learn about how one learns about oneself, and about the cultural, systems, and organizational phenomenon that shape and direct clinical practice and behaviours. To manage the challenge, an IARr has to have a trust in that the objectives stated in the beginning of the AR journey are valid, in this case based on philosophy of caring and midwifery. Such insights elucidate the scholar-practitioner's work when exploring the thoughts, values, and actions, the interiority at the core of the methodology of IAR (Coghlan, 2013). For VN, in this study, the journey was made worthwhile in the shift from the feeling of failure in the early years, to witnessing how tacit caring aspects surfaced in the ward staff in later years, despite the continuation of a strong risk focus in the overall culture of the setting.

\section{Conclusion}

This reflexive account shows that being an IARr while being a novice researcher/ doctoral student is a learning process on how to clinically reflect on and to voice tacit components of care. For the IARr the experience was a struggle, often standing alone at a messy front line, and catalyzing a relational counterbalance to a prevailing medical/technical or risk-focused maternity care system. There is potential for emotional distress and a sense of failure, but there is also potential for delight and unexpected pleasure. Personal preconceptions about what is important to change, and organizational imperatives, blocks and boundaries all play a part in how change will unfold. Shifts in attitudes and behaviours may happen, and tacit change can unravel spontaneously when it is least expected. The strategy used in undertaking this study was influenced by the philosophies of both midwifery care and AR. These included the need to be wholly present during any inter-personal encounter, and to follow the flow, always trusting that the process will, eventually, bring positive (though sometimes unexpected) results.

\section{Acknowledgement}

The authors would like to thank Svante Lifvergren for leading the review process of this article. Should there be any comments/reactions you wish to share, please bring them to the interactive portion (Reader Responses column) of the website: http://arj.sagepub.com. 


\section{Funding}

The author(s) received no financial support for the research, authorship, and/or publication of this article.

\section{References}

2005:12. (2009). Nationella indikatorer för god vård : hälso- och sjukvårdsövergripande indikatorer : indikatorer $i$ Socialstyrelsens nationella riktlinjer [National indicators of good care: health care overall indictors: indicators in the Board's national guidelines]. Stockholm, Sweden: Sverige.Socialstyrelsen.

Adler, N., Shani, A. R., \& Styhre, A. (2004). Collaborative research in organizations: Foundations for learning, change, and theoretical development. Thousand Oaks, CA: SAGE.

Barrett, P. A. (2005). The early mothering project: What happened when the words 'Action Research'came to life for a group of midwives. In P. Reason, \& H. Bradbury (Eds.), Handbook of action research: Concise Paperback Edition (p. 228).

Berg, M., Lundgren, I., Hermansson, E., \& Wahlberg, V. (1996). Women's experience of the encounter with the midwife during childbirth. Midwifery, 12(1), 11-15.

Berg, M., Ólafsdóttir, A., \& Lundgren, I. (2012). A midwifery model of woman-centred childbirth care-In Swedish and Icelandic settings. Sexual \& Reproductive Healthcare, 3(2), 79-87.

Brydon-Miller, M. (2008). Ethics and action research: Deepening our commitment to principles of social justice and redefining systems of democratic practice. In P. Reason, \& H. Bradbury (Eds.), The SAGE handbook of action research: Participative inquiry and practice (pp. 199-210). Los Angeles, London, New Delhi, Singapore: SAGE.

Buber, M., \& Kaufmann, W. (1970). I and thou/Martin Buber: A new translation with a prologue "I and You" and notes by Walter Kaufmann. New York, NY: Simon \& Schuster.

Chandler, D., \& Torbert, B. (2003). Transforming inquiry and action interweaving 27 flavors of action research. Action Research, 1(2), 133-152.

Chiu, L. F. (2006). Critical reflection more than nuts and bolts. Action Research, 4(2), 183-203.

Coghlan, D. (2013). Messy, iterative groping in the swampy lowlands: The challenges of insider scholar-practitioner inquiry. Research in Organizational Change and Development, $21,121-147$.

Coghlan, D., \& Brannick, T. (2009). Doing action research in your own organization. London, England: SAGE.

Dahlberg, K., Dahlberg, H., \& Nyström, M. (2008). Reflective lifeworld research. Lund, Sweden: Studentlitteratur.

Deery, R. (2005). An action-research study exploring midwives' support needs and the affect of group clinical supervision. Midwifery, 21(2), 161-176.

Ellis, C. S., \& Bochner, A. (2000). Autoethnography, personal narrative, reflexivity: Researcher as subject. In N. K. Denzin, \& Y. S. Lincoln (Eds.), Handbook of qualitative research (pp. 733-768). Thousand Oaks: SAGE.

Gadamer, H.-G., Weinsheimer, J., \& Marshall, D. G. (2004). Truth and method. London, England: Continuum.

Hart, E. (1995). Action research for health and social care : A guide to practice. Buckingham, England: Open University Press. 
Heen, H. (2005). About feelings in action research an experiment in first-person inquiry. Action Research, 3(3), 263-278.

Hellström, A., Lifvergren, S., \& Quist, J. (2010). Process management in healthcare: Investigating why it's easier said than done. Journal of Manufacturing Technology Management, 21(4), 499-511.

Heron, J. (1996). Co-operative inquiry : Research into the human condition. London, England: SAGE.

Hoag, B. G., Ritschard, H. V., \& Cooper, C. L. (2002). Obstacles to effective organizational change: The underlying reasons. Leadership \& Organization Development Journal, 23(1), $6-15$.

Holian, R., \& Coghlan, D. (2012). Ethical issues and role duality in insider action research: Challenges for action research degree programmes. Systemic Practice and Action Research, 1-17.

Hunter, B., Berg, M., Lundgren, I., Ólafsdóttir, Ó. Á., \& Kirkham, M. (2008). Relationships: The hidden threads in the tapestry of maternity care. Midwifery, 24(2), 132-137.

Kennedy, H. P. (2000). A model of exemplary midwifery practice: Results of a Delphi study. Journal of Midwifery \& Women's Health, 45(1), 4-19.

Kirkham, M. (2010). The midwife-mother relationship. Basingstoke, England: Palgrave Macmillan.

Lavoie, M., De Koninck, T., \& Blondeau, D. (2006). The nature of care in light of Emmanuel Levinas. Nursing Philosophy, 7(4), 225-234.

Lundgren, I., Karlsdottir, S. I., \& Bondas, T. (2009). Long-term memories and experiences of childbirth in a Nordic context-A secondary analysis. International Journal of Qualitative Studies on Health and Well-being, 4(2), 115-128.

Mander, R., Cheung, N. F., Wang, X., Fu, W., \& Zhu, J. (2010). Beginning an action research project to investigate the feasibility of a midwife led normal birthing unit in China. Journal of Clinical Nursing, 19(3-4), 517-526.

Marshall, J. (2004). Living systemic thinking exploring quality in first-person action research. Action Research, 2(3), 305-325.

McIlveen, P. (2008). Autoethnography as a method for reflexive research and practice in vocational psychology. Australian Journal of Career Development, 17(2), 13-20.

McNiff, J., \& Whitehead, J. (2011). All you need to know about action research. London, England: SAGE.

McVicar, A., Munn-Giddings, C., \& Abu-Helil, C. (2012). Exploring the development of action research in nursing and social care in the UK: A comparative bibliometric review of action research designs in social work (2000-2010). Action Research, 10(1), 79-101.

Meyer, J. (2000). Using qualitative methods in health related action research. $B M J$, $320(7228), 178-181$.

Moore, B. (2007). Original sin and insider research. Action Research, 5(1), 27-39.

Munn-Giddings, C., McVicar, A., \& Smith, L. (2008). Systematic review of the uptake and design of action research in published nursing research, 2000-2005. Journal of Research in Nursing, 13(6), 465-477.

Nugus, P., Greenfield, D., Travaglia, J., \& Braithwaite, J. (2012). The politics of action research:"If you don't like the way things are going, get off the bus". Social Science \& Medicine, 75(11), 1946-1953. 
Nyman, V., Bondas, T., Downe, S., \& Berg, M. (2013). Glancing beyond or being confined to routines: Labour ward midwives' responses to change as a result of action research. Midwifery, 29(6), 573-578.

Nyman, V., Downe, S., \& Berg, M. (2011). Waiting for permission to enter the labour ward world: First time parents' experiences of the first encounter on a labour ward. Sexual \& Reproductive Healthcare, 2(3), 129-134.

O'Connell, R., \& Downe, S. (2009). A metasynthesis of midwives' experience of hospital practice in publicly funded settings: Compliance, resistance and authenticity. Health (London), 13(6), 589-609.

Parkin, P. (2010). Managing change in healthcare: Using action research. London, England: SAGE Publications Ltd.

Reason, P., \& Bradbury, H. (2006). Handbook of action research. London, England: SAGE.

Reason, P., \& Torbert, W. R. (2001). The action turn: Toward a transformational social science. Concepts and Transformation, 6, 1-37.

Russell, K. (2011). Struggling to get into the pool room? A critical discourse analysis of labor ward midwives' experiences of water birth. International Journal of Childbirth, 1(1), $52-60$.

Schön, D. A. (1995). Knowing-in-action: The new scholarship requires a new epistemology. Change: The Magazine of Higher Learning, 27(6), 27-34.

Shani, A. B., Pasmore, W. A., \& Woodman, R. W. (2012). Research in organizational change and development (Vol. 20 [Elektronisk resurs]). Bingley, England: Emerald.

Waterman, H., Tillen, D., Dickson, R., \& de Koning, K. (2001). Action research: A systematic review and guidance for assessment. Health Technol Assess, 5(23), iii-157.

Winter, R., Munn-Giddings, C., \& Atmer, C. (2001). A handbook for action research in health and social care. London, England: Routledge.

\section{Author biographies}

Viola Nyman is a clinical midwife at NÄL-Hospital and a doctoral student at the Institute of Health and Care Sciences, Sahlgrenska Academy, University of Gothenburg, Sweden.

Marie Berg is professor in Health and Care Sciences with focus on Midwifery Science at Institute of Health and Care Sciences, Sahlgrenska Academy, University of Gothenburg, Sweden.

Soo Downe is Professor of Midwifery Studies at the 'Research in Childbirth and Health (ReaCH) group, University of Central Lancashire, Preston, England.

Terese Bondas is professor in Nursing Science, University of Nordland, Bodö, Norway. 